\title{
Image Processing Techniques for Contrast Enhancement with Poor Lighting on Social and Medical Images
}

\author{
Akshay Vartak \\ Asstt.Prof. H.V.P.M.COET, \\ Amravati, India
}

\author{
Vijay Mankar, PhD \\ Dy.Secretary, RBTE, \\ Pune, India
}

\begin{abstract}
Image enhancement is a technique that increases the visual contrast in a designated intensity range. Contrast is an act of distinguishing by comparing differences. Morphological transformation and block analysis are used to detect the background of various social and medical images. Opening by reconstruction method of contrast image transformation can be defined by two operators - opening and closing. The first operator makes use of the information from block analysis, while the second transformation utilizes the opening by reconstruction. The Later is used to define the multi background notion. The complete image processing is being implemented using JAVA simulation model. Quality of image enhancement is assessed by different techniques. In this paper, High performance Computational techniques involving contrast enhancement and noise filtering on various medical, social images are developed using Weber's law. Image quality assessment is compared by different techniques. The values of all the quality assessment parameters are found to be in the standard expected ranges thereby confirming the enhancement of quality of images.
\end{abstract}

\section{Keywords}

Morphological transformation, morphological reconstruction, contrast enhancement, Weber's law, Quality assessment.

\section{INTRODUCTION}

Image enhancement is a challenging research area in image processing. Its principal objective is to process an image so that the result is more suitable than the original image for a specific application. There are several techniques available in this method. Image enhancement is a technique that increases the visual contrast of an image in a designated intensity range or ranges. Visual contrast can be increased by computing image background with morphological transformation and using Weber's law.

In this work, two methodologies to compute the image background are used. Also, some operators are introduced to enhance and normalize the contrast in grey level images and color images with poor lighting. Contrast operators are based on the logarithm function in a similar manner to Weber's law. The logarithm function is used to avoid abrupt changes in lighting. Also, two approximations are used to compute the background in the processed image. There is an analysis by blocks consisted in the first proposal, whereas in the second proposal, the opening by reconstruction is used given its following properties: a) it passes through regional minima, and b) it merges components of the image without considerably modifying other structures. The proposals given in this paper are illustrated with several examples. Once the contrast enhancement is implemented in gray scale it is possible to extend them for colour images. It is to be noted that the objective of image enhancement lies not only in enhancing the quality of the image but also in revealing the objects which are not visible in the original image. All the above implemented methods in gray scale can be also extended to color images by following a standard Segmentation Algorithm procedure.

Finally, this paper is organized as follows. Section II, Weber's Law. Section III, presents morphological Transformations. Section IV presents Block analysis, section V presents opening by reconstruction, section VI ,Image quality assessment, VII, Experimental method, results and comparison. Finally, conclusions are presented in Section VIII.

\section{WEBER'S LAW}

Weber's law expresses a general relationship between a quantity or intensity of something and how much more needs to be added for us to be able to tell that something has been added. Experiments designed to find out such things are called discrimination threshold experiments, because the observer is asked to tell apart, or discriminate, two things that differ by only a slight increment. The discrimination threshold, then, is the smallest detectable increment above whatever was the initial intensity. This general relationship between the initial intensity of something and the smallest detectable increment is exactly what Weber noticed and formalized into Weber's Law.

Weber's law states that, it is the ratio of the difference in maximum to minimum luminance value to the minimum luminance value and it is denoted by $\mathrm{C}$ [2].

$\mathrm{C}=\frac{\mathrm{L}_{\text {max }}-\mathrm{L}_{\text {min }}}{\mathrm{L}_{\text {min }}}$

If $\mathrm{L}=\mathrm{L}_{\text {min }} \Delta \mathrm{L}=\mathrm{L}_{\text {max }}-\mathrm{L}_{\text {min }}$

can be rewritten as

$\mathrm{C}=\frac{\Delta \mathrm{L}}{\mathrm{L}}$

This indicates $\Delta(\log \mathrm{L})$ is in proportionto $\mathrm{C}$; therefore, Weber' s law can be expressed as

$\mathrm{C}=\mathrm{k} \log \mathrm{L}+\mathrm{b} \quad \mathrm{L}>0$

\section{MORPHOLOGICAL}

\section{TRANSFORMATIONS}

Morphology is a technique of image processing based on shape and form of objects. Morphological methods apply a structuring element to an input image, creating an output 
image at the same size. The value of each pixel in the input image is based on a comparison of the corresponding pixel in the input image with its neighbors. By choosing the size and shape of the neighbor, morphological operation can be constructed that is sensitive to specific shapes in the input image. The morphological operations can first be defined on grayscale images and then be expanded to full color images. Morphological operations such as erosion, dilation, opening, and closing. Often combinations of these operations are used to perform morphological image analysis.

\section{BLOCK ANALYSIS}

The method consisting of calculating average between smallest \& largest regional minima has disadvantage that image background is not detected in local way, as result contrast is not correctly enhanced in poor lighting so to compute image background by block analysis, is introduced.

In this analysis, first of all an image will be read as input image and divided into several blocks and from each block, the background will be determined and after applying the Weber's law, an enhanced image will be obtained [9]-[11].

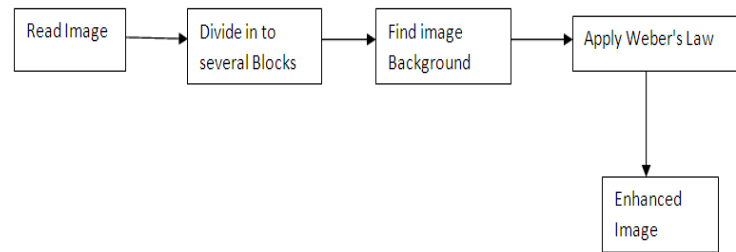

Fig .1. Block diagram of Background Detection by Block Analysis

Let $\mathrm{f}$ be the original image which is subdivided into number of blocks with each block is the sub-image of the original image. For each and every block $\mathrm{n}$, the minimum intensity (mi) and maximum intensity (Mi) values are calculated.

For each analyzed block, maximum (Mi) and minimum (mi) values are used to determine the background criteria $\tau_{i}$ in the following way:

$$
\tau_{i}=\frac{m_{i}+M_{i}}{2} \quad \forall i=1,2, \ldots \ldots n
$$



Fig.2. Background criteria obtained by block analysis.

In the 1-D case, as illustrated in Figure 2, the following expression is obtained:

Once $\tau_{i}$ is calculated, this value is used to select the background parameter associated with the analyzed block. As follows, an expression to enhance the contrast is proposed:

$$
\Gamma \tau_{i}(f)= \begin{cases}k_{i} \log (f+1)+M_{i}, & f \leq \tau_{i} \\ k_{i} \log (f+1)+m_{i}, & \text { otherwise }\end{cases}
$$

Note that the background parameter depends on the $\tau_{i}$ value If $\mathrm{f}<=\tau_{i}$ (dark region), the background parameter takes the value of the maximum intensity (Mi) within the analyzed block, and the minimum intensity (mi) value otherwise. Also, the unit was added to the logarithm function in above equation to avoid indetermination [12]. On the other hand, since grey level images are used in this work, the constant $k_{i}$ in above equation is obtained as follows:

$$
\begin{aligned}
& k_{i}=\frac{255-m_{i}}{\log (256)} \quad \forall_{i}=1,2, \ldots . n \\
& m_{i}= \begin{cases}m_{i}, \quad f>\tau_{i} \\
M_{i}, \quad f \leq \tau_{i}\end{cases}
\end{aligned}
$$

On the other hand, Mi. and mi values are used as background parameters to improve the contrast depending on the $\tau_{i}$ value, due to the background is different for clear and dark regions. Now an image is formed by applying the equation number 13 . Now consider a pixel in this image and the corresponding pixel in original image. Combine them using Weber's law which can be stated as follows. Thus, an enhanced image is formed [13][14].

The more is the number of blocks, the better will be quality of the enhanced image. In the enhanced images, it can be seen that the objects that are not clearly visible in the original image are revealed. As the size of the structuring element increases it is hard to preserve the image as blurring and contouring effects are severe. The results are best obtained by keeping the size of the structuring element as $1(\mathrm{u}=1)$.

\section{OPENING BY RECONSTRUCTION}

This method is similar to block analysis in many ways; apart from the fact that the manipulation is done on the image as a whole rather than partitioning it into blocks.

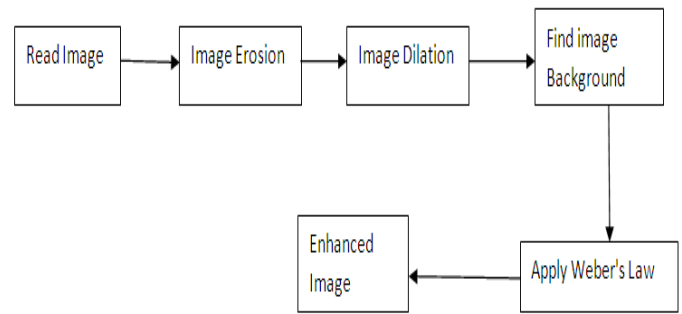

\section{Fig. 3. Block diagram of Background Detection by Erosion \& Dilation}

In a morphological operation, the value of each pixel in the output image is based on a comparison of the corresponding pixel in the input image with its neighbors. By choosing the size and shape of the neighborhood, a morphological operation can be constructed that is sensitive to specific shapes in the input image. Dilation and erosion are two fundamental morphological operations. Dilation adds pixels to the boundaries of objects in an image, while erosion removes pixels on object boundaries. The number of pixels added or removed from the objects in an image depends on the size and shape of the structuring element used to process the image. In 
the morphological dilation and erosion operations, the state of any given pixel in the output image is determined by applying a rule to the corresponding pixel and its neighbors in the input image [15] [16].

In erosion, every object pixel that is touching a background pixel is changed into a background pixel. Dilation adds pixels to the boundaries of objects in an image. In dilation, every background pixel that is touching an object pixel is changed into an object pixel. Note how the function applies the rule to the input pixel's neighborhood and uses the highest value of all the pixels in the neighborhood as the value of the corresponding pixel in the output image. [17] [18].

\section{IMAGE QUALITY ASSESSMENT}

In this section, a comparative evaluation of image quality assessment on enhanced images is done. The quality enhancement was assessed by two methods i.e. subjective method and objective method. In the subjective method, the result in the enhanced image is observed visually, while with the objective methods the quality parameters such as Mean Square Error (MSE), Signal to Noise Ratio (SNR), Peak Signal to Noise Ratio (PSNR), Color Enhancement Factor (CEF), Shannon entropy, wang bovic quality metric, values were calculated within the prescribed range and hence are satisfactory

\section{EXPERIMENTAL METHOD, RESULTS AND DISCUSSION}

Once the contrast enhancement is implemented and established in gray scale images, it can be extended for color images. It is to be noted that the objective of image enhancement lies not only in enhancing the quality of the image but also in revealing the objects which are not visible in the original image. Firstly spatial domain techniques are considered, and then transform domain. All the above implemented methods in gray scale are also extended to color images by following a standard procedure, as described below

Firstly, partition the original image into $R, G$ and $B$ components. Consider a separate component at first (say R), perform the algorithm for the corresponding method i.e. if block analysis is to be implemented for color images, follow the previously mentioned algorithm for block analysis for the $\mathrm{R}$ component. Then repeat the same procedure for $\mathrm{G}$ and $\mathrm{B}$ component. To obtain the enhanced version of the color image as a whole, the $\mathrm{R}, \mathrm{G}$ and $\mathrm{B}$ components are concatenated. This will produce the desired enhanced version of the original image. This procedure can also be successfully applied for Erosion-Dilation method and opening by reconstruction.

A standard segmentation algorithm is used for contrast enhancement which is as follows.

1] Read image data into image array

2] Read each pixel in image array

3] Find minintensity \& maxintensity from its sourrounding pixels

4] Calculate background intensity i.e. mean of max intensity \& min intensity

5] For each sourrounding pixels if pixel intensity is less than background intensity

$\mathrm{k}=(255$-maxintensity $) / \log (256)$;

set pixel intensity $=\mathrm{k} * \log ($ pixel intensity +1$)+\max$ intensity

else $\mathrm{k}=(255-\mathrm{min}$ intensity $) / \mathrm{Math} \cdot \log (256)$

set pixel intensity $=\mathrm{k} * \log ($ pixel intensity +1$)+$ min intensit endif endfor

6] stop endfor



Fig.4.: The architecture of the implemented system

\section{RESULTS AND DISCUSSION}

The enhanced output image is compared with the original image. Both the images are given in fig.5. For various sets of input and output images, the values for MSE ,SNR,PSNR ,CEF, and total time are calculated .They are given in the table1.

\begin{tabular}{|l|l|l|l|l|l|}
\hline $\begin{array}{l}\text { Input } \\
\text { Image }\end{array}$ & MSE & SNR & PSNR & CEF & $\begin{array}{l}\text { Total } \\
\text { Time } \\
(\mathrm{ms})\end{array}$ \\
\hline a1 & 0.50 & 11.36 & 10.67 & 0.283 & 67672 \\
\hline a2 & 0.88 & 8.06 & 10.01 & 0.399 & 4500 \\
\hline a3 & 0.46 & 18.92 & 17.53 & $\begin{array}{l}\text { NAN } \\
\text { (B/W) }\end{array}$ & 22329 \\
\hline
\end{tabular}

Table 1 :- Values obtained from MSE,SNR,PSNR,CEF.

For the same images values of Shannon entropy, SSIM, WBQM are calculated. The values of parameter are given in the table (2),(3),(4).

\begin{tabular}{|c|c|c|c|}
\hline Input Image & $\mathrm{R}$ & $\mathrm{G}$ & $\mathrm{B}$ \\
\hline $\mathrm{a} 1$ & 1.00 & 0.999 & 1.00 \\
\hline $\mathrm{a} 2$ & 1.00 & 1.00 & 1.00 \\
\hline $\mathrm{a} 3$ & 1.00 & 1.00 & 1.00 \\
\hline
\end{tabular}

Table 2 :- Values obtained from Shannon entropy.

\begin{tabular}{|c|c|c|c|}
\hline Input Image & $\mathrm{R}$ & $\mathrm{G}$ & $\mathrm{B}$ \\
\hline $\mathrm{a} 1$ & 0.360 & 0.934 & 0.131 \\
\hline $\mathrm{a} 2$ & 0.353 & 0.354 & 0.238 \\
\hline $\mathrm{a} 3$ & 0.383 & 0.383 & 0.383 \\
\hline
\end{tabular}

Table 3 :- Values obtained from SSIM. 


\begin{tabular}{|c|c|c|c|}
\hline Input Image & $\mathrm{R}$ & $\mathrm{G}$ & $\mathrm{B}$ \\
\hline $\mathrm{a} 1$ & 0.221 & 0.938 & 0.0028 \\
\hline $\mathrm{a} 2$ & 0.125 & 0.185 & 0.075 \\
\hline $\mathrm{a} 3$ & 0.074 & 0.074 & 0.074 \\
\hline
\end{tabular}

Table 4 :- Values obtained from WBQM.

Discussion:- The objective of this research was to detect background and to enhance the quality of grayscale and color images with poor lighting with the help of a standard segmentation algorithm method. The quality enhancement was assessed by two methods i.e. subjective method and objective method. In the subjective method, the result in the enhanced image is observed visually, while with the objective methods the quality parameters such as MSE ,SNR,PSNR, CEF, Shannon entropy, wang bovic quality metric, values were calculated within the prescribed range and hence satisfactory .

\section{CONCLUSION}

This paper presents the method to detect the image background and to enhance the contrast in grey scale and color images with poor lighting. First, block analysis methodology was used to compute an approximation to the background. This proposal was subsequently extended using mathematical morphology operators as well; another methodology based on the use of morphological connected transformations was propounded to detect the image background.

The performances of the proposals provided in this work were illustrated by means of several examples in this paper. Also, Image quality assessment can be performed with different methods such as MSE, SNR , PSNR , SSIM, CEF, Total processing Time, Shannon entropy, Wang-Bovic-QualityMetric (WBQM). The visual inspection of the output image and the calculated values of quality parameters were compared with the standard values of parameters. The subjective as well as the objective methods of assessment of quality enhancement confirm the quality enhancement of original image.

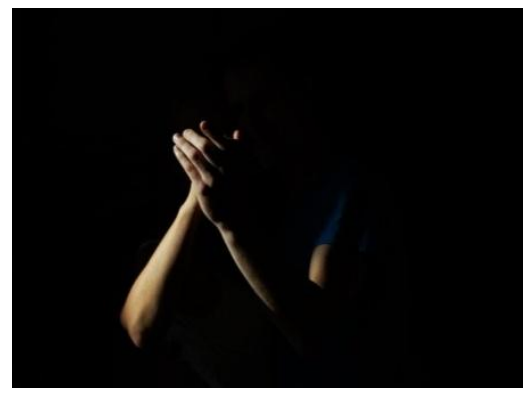

(a)


(b1)

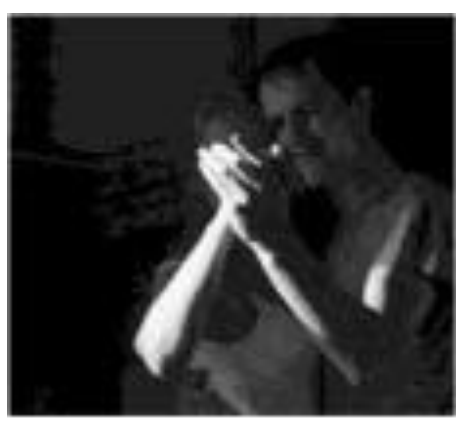

(c1) (b2)

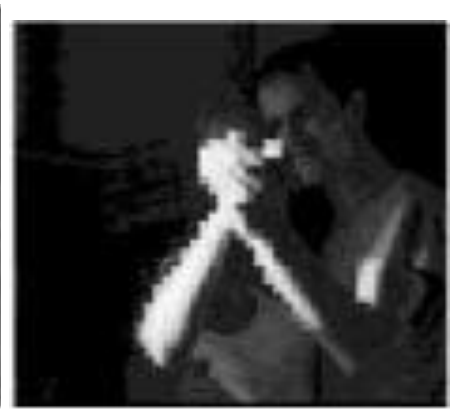

(c2)

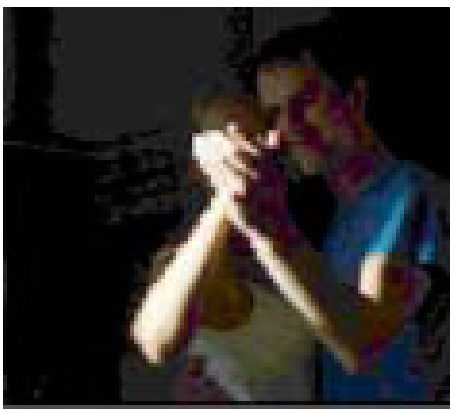

(b3)

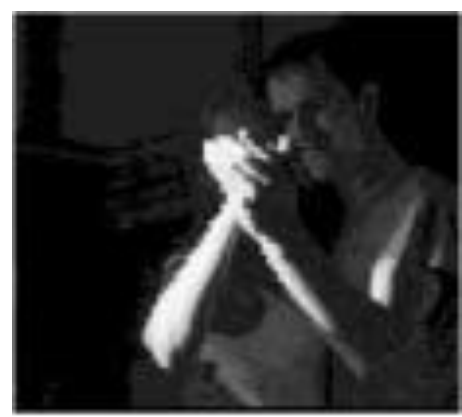

(c3) 




(d1)



(d2)



(d3)

Fig. 5. Image background using the opening by reconstruction with different sizes (a) original image; (b1), (b2), (b3) background images obtained after applying equation (15) with structuring element sizes 10, 30,50; (c1), (c2), (c3),

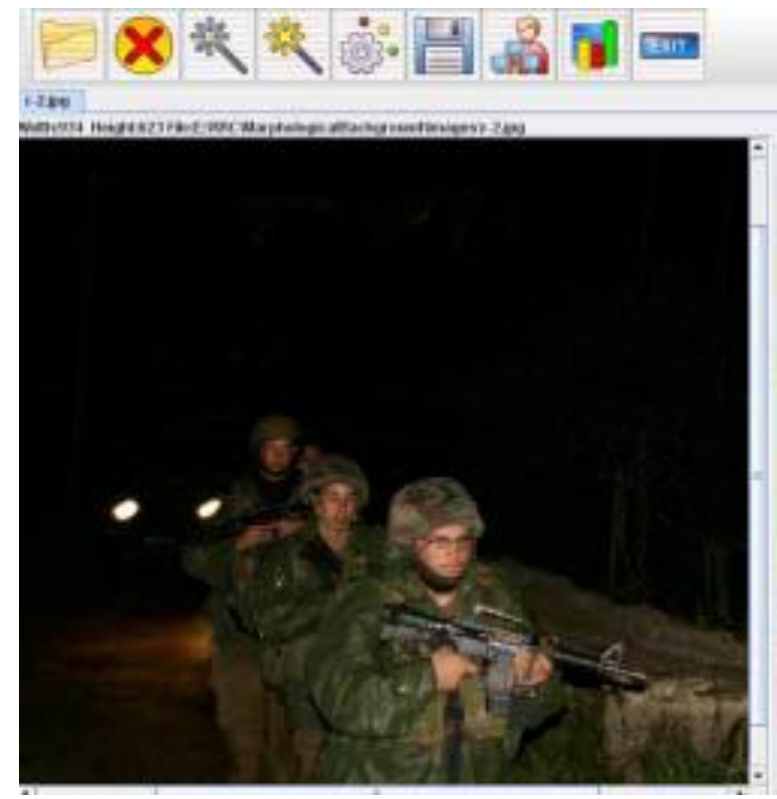

(a1)

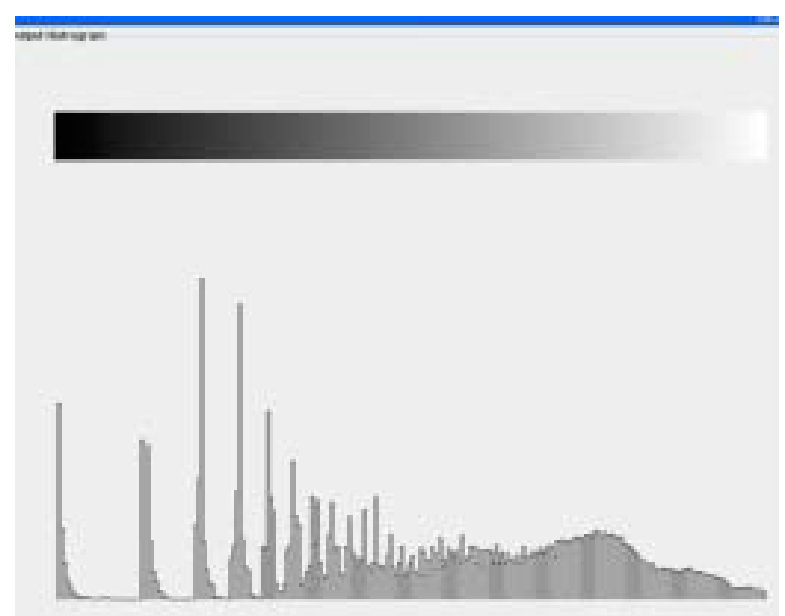

(b1)

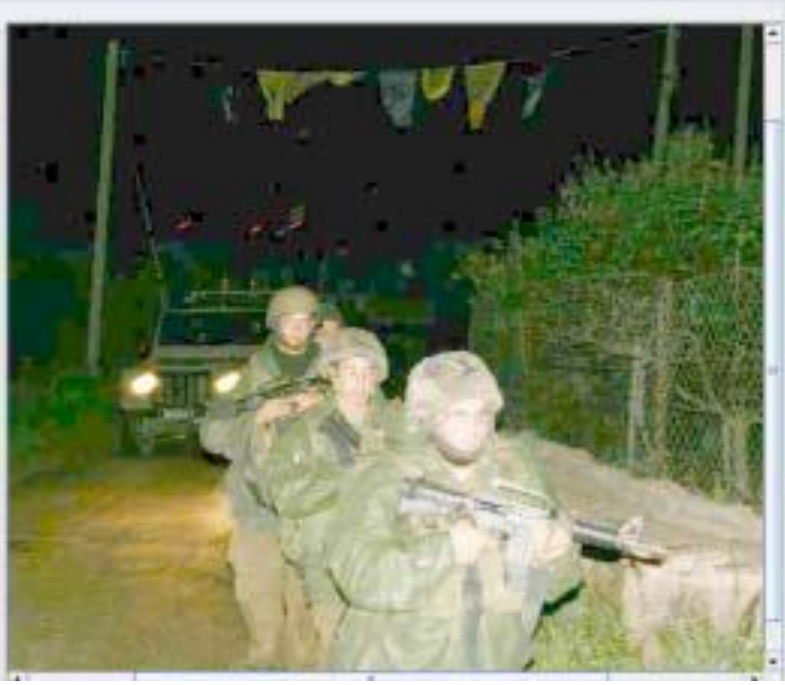

(a2)

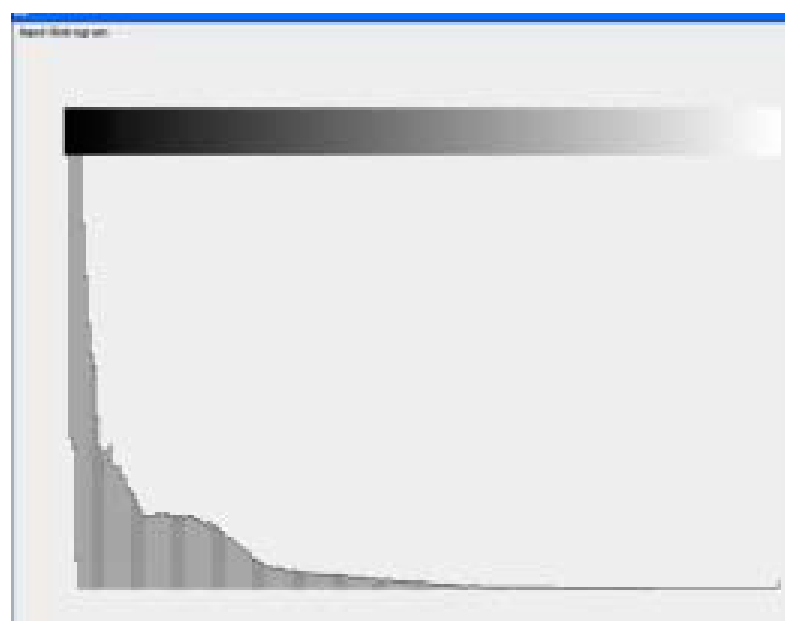

(b2)

Fig.6. Result obtained by proposed segmentation algorithm using JAVA tool .

(a1) Original Color image, (b1) Histogram of Original Image

(a2) Enhanced Color image, (b2) Histogram of enhanced image 




Fig. 7. Result obtained by proposed segmentation algorithm using C Sharp tool

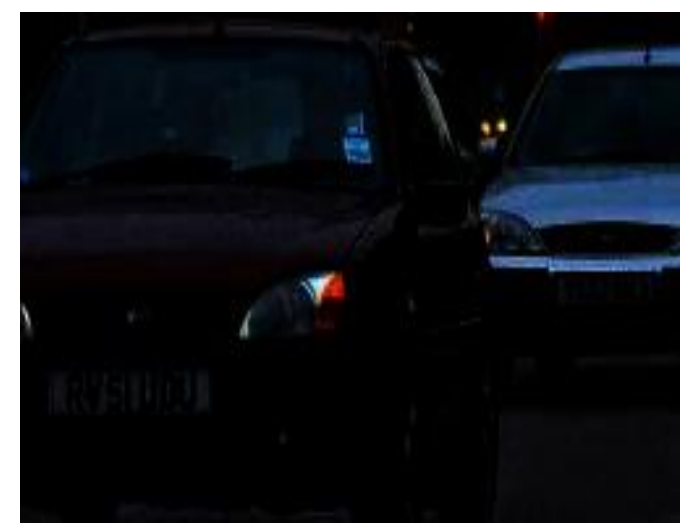

(a1)



(a2)

Fig. 8. (a1) Original Color image, (a2) Enhanced Color image 


\section{REFERENCES}

[1] Humayun K. Sulehria, " Mathematical Morphology Methodology for Extraction of Vehicle Number Plates," International Journal of Computers ,Issue 3, Volume 1, 2007.

[2] Onkar Dabeer,Subhasis Chaudhuri , "Analysis of An Adaptive Sampler Based on Weber's Law",IEEE Transactions on Signal Processing (Impact Factor: 2.81). 05/2011.

[3] Nagarajan Raju,“A Comparison of Contrast Enhancement Techniques in Poor Illuminated Gray Level and Color Images ", International Journal of Computer Applications (0975 - 8887)Volume 25-No.2, July 2011

[4] A.Majumder and S. Irani, "Perception-based contrast enhancement of images," ACM Trans. Appl. Percpt., 4(3): (2007).

[5] S. and R. Manavalan , "Analysis of Background Detection and Contrast Enhancement of MRI Images," International Journal of Computer Applications (0975 - 8887) Volume 36-No.12, December 2011

[6] K.Sreedhar and B.Panlal "Enhancement of Images using Morphological Transformation," International Journal of Computer Science \& Information Technology (IJCSIT) Vol 4, No 1, Feb 2012.

[7] Jianhong Shen "Weber's Law and Weberized TV Restoration," School of Mathematics University of Minnesota Minneapolis, MN 55455, USA.ÀÛÜ

[8] A.I. BEENA and B. ARTHI, "Contrast enhancement in gray-scale images using background approximation by blocks and morphological operations," Oriental Journal of Computer Science\& Technology Vol. 3(1), 69-73 (2010).

[9] Ying-Tung Hsiao, "A Contour based Image Segmentation Algorithm using Morphological Edge Detection ," in IEEE International Conference on (Volume:3 ), 2005

[10] Angélica R. Jiménez-Sánchez, Jorge D.MendiolaSantibañez, "Morphological Background Detection and enhancement of Images with Poor Lighting," IEEE Trans. Image Process. 18(3), pp. 613-623 (2009) .

[11] Soille, P., "Morphological image analysis: principles and applications," Springer Verlag, Berlin (2003).
[12] Xinbo Gao,Image, “Quality Assessment Based on Multiscale Geometric Analysis , "IEEE TRANSACTIONS ON IMAGE PROCESSING, VOL. 18, NO. 7, JULY 2009.

[13] Tinu Alexander John , “ Background Detection of Image using Approximation by Block and Opening by Reconstruction Transformation, " International Conference on Emerging Technology Trends (ICETT) 2011.

[14] Z. Liu, C. Zhang, and Z. Zhang, "Learning-based perceptual image quality improvement for video conferencing," presented at the IEEE Int. Conf. Multimedia and Expo (ICME), Beijing, China, Jul. 2007.

[15] J. Serra and P. Salembier, "Connected operators and pyramids," presented at the SPIE. Image Algebra and Mathematical Morphology, San Diego, CA, Jul. 1993.

[16] A. Toet, "Multiscale contrast enhancement with applications to image fusion," Opt. Eng., vol. 31, no. 5, 1992.

[17] S. Mukhopadhyay and B. Chanda, "A multiscale morphological approach to local contrast enhancement," Signal Process. vol. 80, no. 4, pp. 685-696, 2000.

[18] Erik R. Urbach and Michael H. F. Wilkinson "Efficient 2-D Grayscale Morphological Transformations With Arbitrary Flat Structuring Elements," IEEE TRANSACTIONS ON Image Processing, VOL. 17, NO. 1, January 2008,

\section{AUTHOR PROFILE}

Akshay P. Vartak received his B.E. degree in 1997 in Electronics and Telecommunication from College of Engineering Badnera, M.E. in Digital Electronics from Sipna's College of Engg. \& Technology. Post graduate diploma from CDAC Pune and MBA in Human Resources. He is pursuing $\mathrm{PhD}$ degree in Morphological Image Processing. He is working as an Assistant Professor in HVPM's College of Engg. \& Technology. $\mathrm{He}$ has 12 years of experience in industrial field and 4 years of experience in teaching field as well. He is a member of IETE and ISTE.

Dr. Vijay R. Mankar received his $\mathrm{PhD}$ degree in 2010 from Amravati University. He has more than 25 years of Experience in the field of Engineering \& Technology. He worked as a Head of the Department, Govt. Polytechnic Amravati. He is currently working as a Deputy Secretary RBTE Pune. His research interest includes digital image processing and neural network. 